\title{
Effects of organization and expectancy on recall and recognition
}

\author{
JANE MARANTZ CONNOR \\ State University of New York, Binghamton, New York 18901
}

\begin{abstract}
In two experiments, organization, test type (recall and recognition), and subjects' expectancies of the type of test they would receive (recall or recognition) were varied. It was found that organizational effects may be influenced by both subjects' expectancies of the type of test they will receive and the type of test actually received. Results indicated that subjects' encoding strategies are sensitive to the relationship between the type of material presented and the type of information that they expect to need for the test.
\end{abstract}

One of the predominant views of recall and recognition is that performance on the two types of tasks reflects two qualitatively distinct psychological processes (Anderson \& Bower, 1972; Bahrick, 1970; Kintsch, 1970). It has been proposed that recalling an item involves a stage of memory search or retrieval which is not involved in recognizing an item. According to this view, in recall, subjects first generate a variety of possible responses on the basis of cues provided by either the subject or the experimenter. The subject examines each generated item in turn to determine if he recognizes it as a target item and whether or not to emit it. When a recognition test is employed, the generation or search process is eliminated.

A major source of support for the retrieval distinction between recall and recognition comes from research indicating that increasing list organization facilitates recall substantially but has minimal effects on recognition performance (Kintsch, 1970). A typical pattern of results is that reported by Bruce and Fagan (1970). Bruce and Fagan manipulated list organization by varying the number of categories represented in 42-word lists. High-structure lists contained 6 sevenword categories; low-structure lists contained 42 oneword categories. Distractors for the recognition test were drawn from the same categories in the same proportions as study items. Recognition performance was the same for high- and low-structure lists, whereas recall performance was distinctly superior for highstructure lists relative to low-structure lists. The usual interpretation of these results is that organization facilitates the retrieval process, and retrieval is of

This research was supported by Grant 1R01 MH25344-01 from the National Institute of Mental Health. The comments of an anonymous reviewer were especially helpful. Requests for reprints should be sent to Jane $M$. Connor, Department of Psychology, State University of New York at Binghamton, New York 13901. Part of the results were presented at the meeting of the Midwestern Psychological Association in Chicago, 1975. negligible importance in recognition. More specifically, in the process of writing the word "banana," a recall subject may think of the word "fruit," which would serve as a recall cue for other fruits which appeared on the study list. Since the recognition subject has all the target items (and distractors) before him, the cue does not facilitate his performance.

More recently, Anderson and Bower (1973) have suggested the existence of two distinct encoding processes relevant to recall-recognition differences. They hypothesize that during the study period subjects may tag associative pathways between concepts corresponding to the words shown in the list. The tagging of associative pathways is directly relevant to the retrieval process in recall, since subjects must move from one word concept to another, but the tagging of associative pathways has little to do with recognition performance. Second, subjects tag the concepts corresponding to each presented word with a list tag indicating the occurrence of that word on a particular list. The tagging operation facilitates both recall and recognition. These operations explain the finding that intentional and incidental learning result in equivalent recognition - performance but differential recall performance (Eagle \& Leiter, 1964). Presumably, both intentional and incidental subjects engage in list-tagging, but only intentional subjects tag the associative pathways among word concepts.

If the assumption of two encoding operations is correct, it is of interest to evaluate the extent to which the beneficial effect of list organization on recall is independent of the type of encoding strategy used by the subject and the emphasis placed on the tagging of associative pathways. With respect to recognition performance, it is also of interest whether a strategy that places greater emphasis on list tagging may increase the performance of subjects on a recognition test. (The intentional-incidental learning experiments do not speak to this issue, since incidental learning subjects are engaged in other tasks.) The following experiments were designed to investigate these issues by manipulating 
the type of test subjects were expecting to receive, which would presumably affect the extent to which they engaged in the two types of encoding operations. List organization and the type of test actually received were varied as well. The view of recognition and recall outlined above would predict that the facilitatory effect of list organization on recall either is enhanced for subjects with appropriate expectancies or is unaffected by expectancy. For subjects receiving recognition tests, the overall level of performance may be affected by expectancy, but list organization should have no effect regardless of expectancy. The latter statement is based upon the assumptions that the existence of associative pathways among word concepts and the tagging of these pathways are relevant to recognition performance.

\section{EXPERIMENT 1}

\section{Method}

Subjects. The subjects were 112 undergraduates who participated in the experiment for course credit, in groups of 5 to 15 .

Materials. A pool of 256 words, 8 from each of 32 categories, was selected from the category norms of Battig and Montague (1969). Organized lists consisted of four words from each of eight categories. Eight lists of each type were constructed so that each of the 256 words appeared once on the organized lists and on the unorganized lists. Distractors for the recognition test were taken from the appropriate study list that the subject had not seen previously, so that distractors for the organized lists consisted of a different four words from the same eight categories, and, for the unorganized lists, a single word from the same 32 categories. Distractors for one group of subjects were study words for another group of subjects. The words in the study list and the recognition test were randomly arranged (not blocked by categories) on the page and lists were counterbalanced across subjects and trials.

Procedure. Subjects were given 1 min to study the 32-word list, followed by $1 \mathrm{~min}$ for the arithmetic problems, followed by 2 min to take the test on the list they had just seen. Recall subjects were instructed to write down as many of the words from the preceding lists as they could. A 1 -min rest preceded the next study-test trial. Each subject received a booklet containing the appropriate four sets of study list, arithmetic problems, and test. Subjects received either organized or unorganized lists throughout the session. They were informed that they would receive the same type of test throughout and that they would not be tested more than once on any list. However, on the fourth trial, half of the recall subjects were given recognition tests instead of the recall tests, and vice versa.

\section{Results}

For all recognition scores, mean number correct, corrected for guessing by subtracting the number of false positives from the number of hits, as well as $d^{\prime}$ were computed. Separate analyses of variance were completed for the results of the first three trials and the results of the fourth trial. The analyses were also done separately for subjects receiving recall tests and those receiving recognition tests.

On the first three trials, organization facilitated recall $[\mathrm{F}(1,54)=15.14, \mathrm{MSe}=29.64, \mathrm{p}<.001]$, but had no effect on recognition performance, as measured by d' $[\mathrm{F}(1,54)=1.83$, MSe $=.52$, n.s. $]$ and by number correct $[\mathrm{F}(1,54)<1, \mathrm{MSe}=21.51]$. No effect of trials was found in any of the analyses $[F(1,54)=2.03$, $\mathrm{MSe}=10.56$, n.s., for recall; $\mathrm{F}(1,54)=1.82, \mathrm{MSe}=9.41$, n.s., for recognition number correct; $F(1,54)=1.57$, MSe $=.15$, n.s., for $\left.\mathrm{d}^{\prime}\right\}$. The effect of organization did not vary as a function of trial number; none of the interactions of trial with organization were significant.

The results of the fourth trial are shown in Table 1. For recognition performance, the type of test expected and the type of list studied had no effect on number correct or $\mathrm{d}^{\prime}$. (MSe was 19.90 for number correct and .44 for $\mathrm{d}^{\prime}$. The $\mathrm{F}$ values for the tests of main effects and interactions ranged from less than 1 to 1.5 , with 1 and 54 degrees of freedom).

Looking at recall performance, subjects expecting recall scored higher than subjects expecting recognition $[F(1,54)=21.85, \mathrm{MSe}=28.03, \mathrm{p}<.001]$, and subjects studying categorized lists scored higher than subjects studying noncategorized lists $[F(1,54)=6.89, p<.01]$. The relative superiority of categorized lists over noncategorized lists was the same for both types of expectancy $[F(1,54)<1]$.

\section{Discussion}

The results of this experiment are consistent with the model of recognition and recall outlined in the introduction. The overall performance of recall subjects was markedly affected by expectancy, indicating that

Table 1

Mean Number of Words Correct and $d^{\prime}$ on Fourth Trial as a Function of Organization, Test Received, and Test Expected

\begin{tabular}{|c|c|c|c|c|}
\hline \multirow[b]{3}{*}{ Test Received } & \multicolumn{4}{|c|}{ Type of List } \\
\hline & \multicolumn{2}{|c|}{ Noncategorized } & \multicolumn{2}{|c|}{ Categorized } \\
\hline & $\begin{array}{l}\text { Number } \\
\text { Correct }\end{array}$ & $\mathrm{d}^{\prime}$ & $\begin{array}{l}\text { Number } \\
\text { Correct }\end{array}$ & $d^{\prime}$ \\
\hline & \multicolumn{4}{|c|}{ Experiment 1} \\
\hline \multicolumn{5}{|l|}{ Recognition $\mathbf{a}, \mathbf{b}$} \\
\hline Expecting Recall & 20.64 & 2.22 & 21.71 & 2.19 \\
\hline Expecting Recognition & 22.57 & 2.12 & 21.64 & 2.02 \\
\hline \multicolumn{5}{|l|}{ Recall ${ }^{\mathrm{c}}$} \\
\hline Expecting Recall & 13.93 & & 18.29 & \\
\hline \multirow[t]{2}{*}{ Expecting Recognition } & 6.43 & & 12.57 & \\
\hline & \multicolumn{4}{|c|}{ Experiment 2} \\
\hline \multicolumn{5}{|l|}{ Recognition ${ }^{d, e}$} \\
\hline Expecting Recall & 24.33 & 2.36 & 28.06 & 3.15 \\
\hline Expecting Recognition & 26.11 & 2.72 & 25.61 & 2.58 \\
\hline \multicolumn{5}{|l|}{ Recallf $^{f}$} \\
\hline Expecting Recall & 11.72 & & 15.39 & \\
\hline Expecting Recognition & 11.50 & & 11.61 & \\
\hline
\end{tabular}

(a) least significant difference $=3.38$ for number correct, $p<.05$. (b) least significant difference $=.50$ for $d^{\prime}, p<.05$. (c) least significant difference $=4.02, p<.05$. (d) least significant difference $=2.71$ for number correct, $p<.05$. (e) least significant difference $=.43$ for $d^{\prime}, p<.05$. (f) least significant difference $=2.51, p<.05$. 
subjects expecting recognition placed less emphasis on the tagging of associative pathways than did those expecting recall. List organization facilitated recall to a similar extent for both subjects expecting recall and those expecting recognition. The lack of interaction between expectancy and list organization for recall subjects implies that the locus of the list-organization effect in the experiment was at the retrieval rather than at the encoding stage. If list organization also had an effect on encoding strategy, one would have expected an interaction with expectancy on recall performance.

The recognition data are also consistent with the Anderson and Bower (1973) model. The existence of appropriate occurrence information, which is assumed to be the sole determinant of recognition performance, was unaffected by either list organization or expectancy. From the lack of any expectancy effect it must be inferred that subjects expecting recognition are not any more efficient or successful at tagging occurrence information than are subjects expecting recall, who are additionally tagging associative pathways. The lack of an effect of list organization on recognition performance is a replication of previous work (cf. Kintsch, 1970).

It has been argued, however, that organization will facilitate recognition when sufficiently strong manipulations are introduced (Mandler, 1972). In Mandler's research (Mandler, 1972; Mandler, Pearlstone, \& Koopmans, 1969), subjects sorted a set of unrelated words into a number of categories of their own definition. The subjects thus imposed their own organizational structures on the list. In Experiment 1, in contrast, the organizational structure was defined by the experimenter and may not have been sufficiently flexible for subjects to adapt their encoding strategies to the type of list given.

A further complication in the study was that both the set of study words and the set of distractors varied for organized and unorganized lists. Since the type and number of distractors used in the recognition test are clearly critical to recognition performance (Anisfeld \& Knapp, 1968), it would be preferable to keep the set of distractors constant across the levels of the organization manipulation. Although the set of distractors was balanced across subjects in the organized and unorganized conditions, there may have been a greater degree of confusability for subjects in the organized conditions than in the unorganized condition. To alleviate this difficulty and to permit greater flexibility in encoding strategies, in Experiment 2, all subjects received categorized lists and all recognition subjects received identical tests. Degree of organization was varied either by arranging words randomly on the page or by blocking words by category. To the extent that subjects expecting recall may spend more time tagging associative pathways than subjects expecting recognition, the blocked arrangement would allow them to do so more efficiently.

\section{EXPERIMENT 2}

\section{Method}

Subjects. There were 144 undergraduates who participated in this experiment. They were tested in groups ranging in size from 5 to 15 .

Materials. Only the lists containing four words from each of eight categories that were constructed for Experiment 1 were used in Experiment 2. Half the subjects received study lists that were blocked by category throughout the experiment, while the other half received lists in which the order of words in a list was randomly determined. The distractors in the recognition test were identical for blocked and random groups, and consisted of four words from each of the same eight categories as appeared in the study list, arranged randomly on the page. The procedure and design were otherwise the same as in Experiment 1. On Trial 4, half the subjects who were expecting a recall test received a recognition test, and vice versa.

\section{Results}

The results of the first three trials in Experiment 2 were parallel to the results of the first three trials in Experiment 1. Blocking facilitated recall $[F(1,70)=10.84, \quad M S e=23.35, \quad p<.001], \quad$ but had no effect on recognition as measured by number correct $[\mathrm{F}(1,70)=1.55, \mathrm{MSe}=22.86$, n.s. $]$, or by $\mathrm{d}^{\prime}$ $[F(1,70)<1, \mathrm{MSe}=.49]$. The effect of trials and the interaction of Trials by Blocking were not significant for recall or the two recognition measures.

The results of Trial 4 are shown in Table 1. For recognition subjects, the interaction of Blocking by Type of Test Expected was significant for both dependent measures $[\mathrm{F}(1,68)=9.49, \quad \mathrm{MSe}=.41$, $\mathrm{p}<.01$, for $\mathrm{d}^{\prime}$, and $\mathrm{F}(1,68)=5.01, \mathrm{MSe}=16.57$, $p<.05$, for number correct] . Subsequent tests indicated that blocking facilitated recognition performance only for subjects expecting recall (see Table 1). As measured by $\mathrm{d}^{\prime}$, recognition performance in blocked lists was better for subjects expecting recall than for subjects expecting recognition $[\mathrm{F}(1,68)=7.03, \quad \mathrm{MSe}=.42$, $\mathrm{p}<.01]$.

For recall subjects, the interaction of Blocking by Type of Test Expected was also significant $[\mathrm{F}(1,68)=4.04, \mathrm{MSe}=14.14, \mathrm{p}<.05]$. Blocking facilitated recall for subjects expecting recall $[\mathrm{F}(1,68)=8.57$, $\mathrm{p}<.01]$, but not for subjects expecting a recognition test $[\mathrm{F}(1,68)<1]$. Performance on blocked lists was superior for subjects expecting recall, rather than recognition $[F(1,68)=9.09, p<.01]$, but expectancy did not affect recall performance on randomly arranged lists $[F(1,68)<1]$.

\section{Discussion}

The results of Experiment 2 provide some interesting contrasts with the results of Experiment 1. Among recall subjects in Experiment 2, blocking facilitated performance only when subjects were expecting recall. In Experiment 1, list organization, as varied by the number of categories on a list, had a facilitatory effect 
on recall, regardless of expectancy. This suggests that the locus of the organization effect may have been different in the two experiments. Blocking may have facilitated recall because of the greater ease with which subjects can tag associative pathways while studying a blocked rather than a randomly arranged list. List structure (manipulated by number of categories) may have facilitated recall because of the greater number of associative pathways that exist among words on a categorized list as opposed to a noncategorized list.

The implications of the recognition results in Experiment 2 for the model described in the introduction are less clear. Blocking facilitated recognition only for subjects expecting recall, that is, for subjects who were encouraged to encode the relationships among the word clusters. For some reason, the tagging of associative pathways facilitated recognition when such tagging was easy to accomplish, in other words, in the blocked list. Possibly, such tagging, when combined with a blocked list, encourages the subjects to rehearse the target words in groups during the study period, which results in a strengthening of the list-occurrence information for the words within the group. The results of the two experiments together indicate that subjects have greater difficulty generating efficient encoding strategies for recognition tasks. One problem is that the type of information required to do well on a recognition test is determined by the set of distractors. However, even with a general knowledge of the type of distractors that will be encountered, as was the case in these experiments, such strategies appear to be difficult to devise.

\section{CONCLUSIONS}

As a whole, the results of these experiments suggest two important methodological considerations. First, studies comparing recognition and recall over a series of tests should contain adequate control for the different encoding strategies subjects may adopt as a function of the type of test they are expecting. Second, the way in which list organization is varied (categorization, blocking, etc.) may affect the pattern of results obtained in interaction with other factors. It is particularly important that the degree of similarity between the set of target items and the set of distractors be similar for the two types of lists. Many of the studies on the effect of list organization and recognition which have found negative results have confounded organization in the study set with organization in the distractor set (e.g., Bruce \& Fagan, 1970; Kintsch, 1968). Other studies that have manipulated list organization by varying the spatial or temporal arrangement of study items have found effects on recognition (e.g., Bower, Clark, Lesgold, \& Winzenz, 1969; Bower \& Winzenz, 1969). List organization appears to be a multidimen. sional rather than a unidimensional factor.

\section{REFERENCES}

ANDerson, J. R., \& Bower, G. H. Recognition and retrieval processes in free recall. Psychological Review, 1972, 79, 97-123.

ANDERSON, J. R., \& Bower, G. H. Human associative memory, Washington, D.C: V. H. Winston, 1973.

ANisfeld, M., \& KNAPP, M. Association, synonymity and directionality in recognition. Journal of Experimental Psychology, 1968, 77, 171-179.

BAHRICK, H. P. Two-phase model for prompted recall. Psychological Review, 1970, 77, 215-222.

Battig, W. F., \& Montague, W. E. Category norms for verbal items in 56 categories. Journal of Experimental Psychology Monographs, 1969, 80.

Bower, G. H., Clark, M., Lesgold, A. M., \& Winzenz, D. Hierarchial retrieval schemes in recall of categorized word lists. Joumal of Verbal Learning and Verbal Behavior, 1969, 8, 323-343.

Bower, G. H., \& Winzenz, D. Group structure, coding and memory for digit series. Journal of Experimental Psychology Monographs, 1969, 80(No. 2, Part 2), 1-17.

Bruce, D., \& FaGAN, R. I. More on the recognition and free recall of organized lists. Joumal of Experimental Psychology, 1970, 85, 153-154.

EAgLe, M., \& Leiter, E. Recall and recognition in intentional and incidental learning. Journal of Experimental Psychology, 1964, 68, 58-63.

KinTsCH, W. Recognition and free recall of organized lists. Journal of Experimental Psychology, 1968, 78, 481.487.

KinTsCh, W. Models for free recall and recognition. In D. A. Norman (Ed.), Models of human memory. New York: Academic Press, 1970.

MANDLER, G. Organization and recognition. In E. Tulving \& W. Donaldson (Eds.), Organization of memory. New York: Academic Press, 1972.

Mandler, G., Pearlstone, Z., \& Koopmans, H. S. Effects of organization and semantic similarity on recall and recognition. Joumal of Verbal Learning and Verbal Behavior, 1969, 8, 410-423.

(Received for publication October 26, 1976; revision accepted January 13,1977 .) 